\title{
Renormalization Group Approach to Causal Bulk Viscous Cosmological Models
}

\author{
J. A. Belinchónf \\ Grupo Inter-Universitario de Análisis Dimensional. \\ Dept. Física ETS Arquitectura UPM Av. Juan de Herrera 4 Madrid 28224 España \\ T. Harkol] \\ Department of Physics, The University of Hong Kong, \\ Pokfulam Road, Hong Kong, P. R. China. \\ M. K. Makf \\ Department of Physics, The Hong Kong University of Science and Technology, \\ Clear Water Bay, Hong Kong, P. R. China.
}

(Dated: March 26, 2002)

\begin{abstract}
The renormalization group method is applied to the study of homogeneous and flat FriedmannRobertson-Walker type Universes, filled with a causal bulk viscous cosmological fluid. The starting point of the study is the consideration of the scaling properties of the gravitational field equations, of the causal evolution equation of the bulk viscous pressure and of the equations of state. The requirement of scale invariance imposes strong constraints on the temporal evolution of the bulk viscosity coefficient, temperature and relaxation time, thus leading to the possibility of obtaining the bulk viscosity coefficient-energy density dependence. For a cosmological model with bulk viscosity coefficient proportional to the Hubble parameter, we perform the analysis of the renormalization group flow around the scale invariant fixed point, therefore obtaining the long time behavior of the scale factor.
\end{abstract}

PACS numbers: 98.80.Hw, 98.80.Bp, 04.20.Jb

\footnotetext{
*Electronic address: abelcal@ciccp.es

${ }^{\dagger}$ Electronic address: tcharko@hkusua.hku.hk

${ }^{\ddagger}$ Electronic address: mkmak@vtc.edu.hk
} 


\section{INTRODUCTION}

The renormalization group $(\mathrm{RG})$ has been proved to be a powerful tool in statistical physics, quantum field theory, critical phenomena and chaotic dynamic [1], 3]. The essence of the renormalization group method is to extract structurally stable features of a system, which are insensitive to details. Many physical systems in field theories, polymers etc. exhibit universal scaling functions and critical exponents in the limit $\Lambda / \xi$, where $\Lambda$ is some ultraviolet cut-off and $\xi$ is a temperature-dependent correlation length. The RG group method is the main tool with which to elucidate this universal behavior. On the other hand, from a purely mathematical point of view, the $\mathrm{RG}$ is regarded as a means of asymptotic analysis. Long-time asymptotics of non-linear partial differential equations using RG ideas have been studied recently by Bricmont, Kupiainen and Lin [4 and by Chen, Goldenfeld and Oono [5]. In this formulation, the RG transformation is the integration of the equation up to a finite time, followed by a rescaling of the dependent and independent variables. Therefore the problem at infinite time is reduced to the problem at finite time. A fixed point of the RG transformation corresponds to a scale-invariant solution of the differential equation, and the long-time behavior of the solution can be obtained by studying the flows around fixed points. The RG method for reduction of evolution equations has been formulated in terms of the notion of invariant manifolds in [6], and it has been shown that the perturbative RG method constructs invariant manifolds successively, as the initial value of the evolution equation.

The RG method has been also intensively applied in the study of cosmological models and of the critical behavior of the gravitational collapse of a radiation fluid. Koike, Hara and Adachi [7] presented a scenario based on the renormalization group that can explain the universality and scaling observed in a numerical study of gravitational collapse of radiation fluid. This approach for understanding and analyzing critical behavior in the gravitational collapse has been generalized, to a general framework, for perfect fluids with pressure proportional to density and with arbitrary $\gamma$ [8]. Critical phenomena occur for $1<\gamma<1.88$ and the uniqueness of the relevant mode around a fixed point is established by Liapunov analysis.

The averaging hypothesis tacitly assumed in standard cosmology was discussed by using RG methods by Carfora and Piotrkowska [9]. A critical behavior, which can be related to 
the formation of sheet-like structures in the Universe, is obtained, and explicit expressions for the normalized Hubble constant and for the scale dependence of matter distribution are also provided. The observable scaling properties in Newtonian cosmology have been considered in [10]. The quantitative scaling properties have been analyzed by calculating the characteristic exponents around each fixed point, thus leading to the possibility of a fractal structure of the Universe beyond a horizon scale. The RG method has been applied to the Einstein equations in cosmology by Iguchi, Hosoya and Koike [1], who carried out a detailed analysis of renormalization group flows in the vicinity of the scale invariant fixed point in the spherically symmetric and inhomogeneous dust filled Universe. The first order solution of long-wavelength expansion of the Einstein equations has been improved in [12]. By assuming that the RG transformations have the property of Lie group, the secular divergence caused by the spatial divergence terms can be regularized and absorbed to the background seed metric. The renormalized metric describes qualitatively quite well the behavior of the gravitational collapse in the expanding Universe. The back reaction effect due to the inhomogeneities has been incorporated into the framework of the cosmological perturbations, using the RG method, by Nambu [13]. The RG equations, which determines the dynamics of the spacetime, have been derived in a gauge invariant manner.

The basic techniques and ideas for the construction of RG theories for the study of non-equilibrium processes in inflationary and semiclassical cosmology and stochastic gravity have been recently reviewed in [14]. The complete study of the flow of the renormalization group in the Einstein-Hilbert truncation of quantum gravity in four dimensions and the classification of the RG trajectories have been done in [15]. Cosmologies with a timedependent Newton constant and a cosmological constant have been investigated, from the RG point of view, by Bonnano and Reuter [16]. The scale dependence of $G$ and $\Lambda$ is governed by a set of RG equations, which are coupled to the Einstein equations in a consistent way. This model could explain the data from recent observations of red-shift of Type Ia Supernovae without introducing a quintessence field. The cosmological implications of the running of the cosmological constant and whether the scaling dependences of $G$ and $\Lambda$ spoil primordial nucleosynthesis have been discussed, using RG techniques, in [17].

It is the purpose of the present paper to apply the RG group techniques to the analysis of the bulk viscous cosmological models.

Dissipative processes are supposed to play a fundamental role in the evolution and dy- 
namics of the early Universe. Over thirty years ago Misner [18 suggested that the observed large scale isotropy of the Universe is due to the action of the neutrino viscosity, which was effective when the Universe was about one second old. Bulk viscosity may arise in different contexts during the evolution of the early Universe. Some physical processes involving viscous effects are the evolution of cosmic strings, the classical description of the (quantum) particle production, interaction between matter and radiation, quark and gluon plasma, interaction between different components of dark matter etc. [19].

The first attempts at creating a theory of relativistic dissipative fluids were those of Eckart 20] and Landau and Lifshitz [21]. These theories are now known to be pathological in several respects. Regardless of the choice of equation of state, all equilibrium states in these theories are unstable and in addition signals may be propagated through the fluid at velocities exceeding the speed of light [22]. The problems arise due to the first order nature of the theory, i.e., it considers only first-order deviations from the equilibrium leading to parabolic differential equations, and hence to infinite speeds of propagation for heat flow and viscosity, in contradiction with the principle of causality. Infinite propagation speeds already constitute a difficulty at the classical level, since one does not expect thermal disturbances to be carried faster than some (suitably defined) mean molecular speed. Conventional theory is thus applicable only to phenomena which are quasi-stationary, i.e., slowly varying on space and time scales characterized by mean free path and mean collision time [23]. This is inadequate for many situations in high-energy astrophysics and relativistic cosmology involving steep gradients or rapid variations. The deficiencies can be traced to the fact that the conventional theories (both classical and relativistic) make overly restrictive hypothesis concerning the relation between the fluxes and densities of entropy, energy and particle number.

A relativistic second-order theory was found by Israel [22] and developed by Israel and Stewart [23, into what is called transient or extended irreversible thermodynamics. In the second order theory the deviations from equilibrium (bulk stress, heat flow and shear stress) are treated as independent dynamical variables leading to a total of 14 dynamical fluid variables to be determined. However, Hiscock and Lindblom [24] and Hiscock and Salmonson 25] have shown that most versions of the causal second-order theories (the socalled truncated models) omit certain divergence terms. When these terms are added to the evolution equation of the bulk viscous pressure, one obtain the full causal theory, with well 
behaved solutions for all times. Causal bulk viscous thermodynamics has been extensively used for describing the dynamics and evolution of the early Universe and some astrophysical process [26, 42].

The fact that the Universe has some hierarchical structure, and that the scale invariant solutions of the gravitational field equations plays an important role is well known [11]. The homogeneous Einstein equations are scale invariant. Using this property, we apply the RG method to study the long-time asymptotics of the general relativistic field equations, describing the evolution of a homogeneous causal bulk viscous fluid filled Universe. For the RG method we closely follow the approach presented in [11].

The renormalization group approach shows the particular role played by the exponent $s$ of the density in the equation of state of the bulk viscous coefficient. The gravitational field equations are invariant under scale transformations only for the value $s=1 / 2$ of the

exponent. Hence this method provides an effective and efficient tool for investigating general properties of cosmological models.

The present paper is organized as follows. In Section II we give a brief and formal presentation of the renormalization group method and its applications to the study of the asymptotics of differential equations. The scaling properties of the basic equations of the causal thermodynamics in a homogeneous space-times are investigated in Section III, and their implications on the equation of state of the cosmological causal bulk viscous fluid are considered. The flow in the RG around the fixed points of the renormalization group equations is analyzed in Section IV. Finally, in Section V we discuss and conclude our results.

\section{RENORMALIZATION GROUP AND DIFFERENTIAL EQUATIONS}

In this Section we briefly review, from a formal and abstract mathematical point of view, the formalism of renormalization group, as applied to the study of systems of ordinary or partial differential equations. The presentation closely follows [国] and [8], and for further details and specific applications the reader is referred to these papers.

The basic idea in the RG formalism is to consider the time evolution of initial data as a flow of a renormalization group transformation. Suppose we are interseted in the time evolution of $n$ unknown functions $y=\left(y_{1}, y_{2}, \ldots, y_{n}\right)$, which are real-valued functions of the 
time $t$ and of a spatial coordinate $x$, and satisfy a system of partial differential equations

$$
F\left(y, \frac{\partial y}{\partial t}, \frac{\partial y}{\partial x}, t, x\right)=0 .
$$

The time evolution of the system (1) is determined once one specifies the values $y(t, \cdot)$ at the initial time $t=1$. The space of functions $y(t, \cdot)$ at a fixed time is called the phase space $\Gamma$ of the system, and assume it is a real vector space. As a first step in introducing the renormalization group transformation, we define the scaling transformation $S(L, \vec{\alpha}, \beta)$, which depends on the real parameters $L \in R, \vec{\alpha}=\left(\alpha_{1}, \alpha_{2}, \ldots, \alpha_{n}\right) \in R, \beta \in R$ [B]]:

$$
S(s, \vec{\alpha}, \beta): y_{i}(t, x) \rightarrow y_{i}^{(L, \vec{\alpha}, \beta)}(t, x) \equiv e^{\alpha_{i} L} y_{i}\left(e^{-L} t, e^{-\beta s} x\right) .
$$

If $y$ is a solution of the Eq. (11), and if with a suitable choice of the constants $\vec{\alpha}, \beta$ and for an arbitrary scale parameter $L$ (close to zero), the new function $y^{(L, \vec{\alpha}, \beta)}(t, x)$ is also a solution of Eq. (11), then the system (11) is said to be invariant under the scaling transformation (2). In the following we denote, for simplicity, $y_{i}^{(s, \vec{\alpha}, \beta)}$ by $\stackrel{(L)}{y}_{i}$, and assume that generally $L>0$.

The renormalization group transformation $\Re_{s}$ is defined as a transformation acting on the phase space $\Gamma$ of the system (11) [8]:

$$
\Re_{L}: Y_{i}(\xi)=y_{i}(1, \xi) \rightarrow \stackrel{(L)}{Y_{i}}(\xi)=\stackrel{(L)}{y_{i}}(1, \xi)=e^{\alpha_{i} L} y_{i}\left(e^{-L}, e^{-\beta L} \xi\right) .
$$

More exactly, $\stackrel{(L)}{Y}$ is given by developing the initial data $y(1, x) \equiv Y(x)$ from $t=1$ to $t=e^{-L}$ by Eq. (四), and then rescaling the spatial coordinate $x$ and the unknown functions $y_{i}$. Because of the invariance under scaling, $\Re_{L}$ forms a semi-group with the property $\Re_{L_{1}+L_{2}}=\Re_{L_{2}} \circ \Re_{L_{1}}$. From this property one can find the infinitesimal generator $\dot{\Re}_{\text {of }} \Re_{L}$, given by the derivative of $\Re_{L}$ with respect to $L$ at $L=0, \dot{\Re} \equiv \lim _{L \rightarrow 0} \frac{\Re_{s}-1}{L}$, leading to $\Re_{L}=\exp (L \dot{\Re})$.

A fixed point of the renormalization group $\left\{\Re_{L} \mid L \in R\right\}$ is a point $Y^{*}$ in $\Gamma$ satisfying the equation $\Re_{L}\left(Y^{*}\right)=Y^{*}$ for all $L>0$. Equivalently, it can also be defined as $\dot{\Re}\left(Y^{*}\right)=0$. A function $y$ is called self-similar with parameters $(\vec{\alpha}, \beta)$ if it satisfies $y(t, x)=y^{(L, \vec{\alpha}, \beta)}(t, x)$. Each self-similar solution $y_{s s}$ of Eq. (1) with parameters $(\vec{\alpha}, \beta)$ is related to a fixed point $Y^{*}$ of $\Re_{L}$ by $y_{s s}(t, x)=(-t)^{\alpha} Y^{*}\left[x(-t)^{-\beta}\right]$ [ $]$.

As a next step we introduce the tangent map of $\Re_{L}$ at $Y$. It is defined by $T_{L, Y} \equiv$ $\lim _{\epsilon \rightarrow 0} \frac{\Re_{L}(Y+\epsilon K)-\Re_{L}(Y)}{\epsilon}$. For a fixed $L$ and $Y, T_{L, Y}$ is a linear operator, related to the renormalization group by the following formal relation: $\Re_{L}(Y+K)=\Re_{L}+T_{L, Y}(K)+O(K)^{2}+\ldots$ 
At a fixed point $Y^{*}$ of $\Re_{L}$, the tangent map is $T_{L, Y^{*}}=T_{L}(K) \equiv \lim _{\epsilon \rightarrow 0} \frac{\Re_{L}\left(Y^{*}+\epsilon K\right)-\Re_{L}\left(Y^{*}\right)}{\epsilon}$. The tangent map at the fixed point forms a semi-group. In terms of the infinitesimal generator $\dot{T}=\lim _{L \rightarrow 0} \frac{T_{L}-1}{L}$, the tangent map can be represented as $T_{L}=\exp (L \dot{T})[8]$.

The flow of the renormalization group transformation near the fixed point $Y^{*}$ is determined by the eigenmodes of $\dot{T}$, with eigenvalues $k \in \mathbb{R}, \mathbb{C}$, defined as $Y^{*} K=k K$. A mode with $\operatorname{Re} k>0$ is called a relevant mode, and it is tangent to a flow diverging from $Y^{*}$. A mode with Re $k<0$ is tangent to a flow converging to the fixed point. Modes with $\operatorname{Re} k=0$ are called marginal. If there is a finite number of relevant modes, or no relevant mode, then the long-time behavior of nonlinear partial equations can be predicted from the study of the perturbations around the fixed point.

To make the abstract approach presented above more clearer from the point of view of the physical applications, we discuss the RG method for an equation of the type [1]

$$
\dot{y}=y^{\prime \prime}+F\left(y, y^{\prime}, y^{\prime \prime}\right)
$$

where a dot and a prime denote the derivatives with respect to the variable $t$ and $x$, respectively. We are interested in the asymptotics of the solution of the form $y(t, x) \sim$ $t^{-\alpha / 2} f\left(t^{-1 / 2} x\right)$, as $t \rightarrow \infty$. The RG methods transforms the problem of large time limit into an iteration of a fixed time problem, followed by a scaling transformation. The scaleinvariant solution emerges then as a fixed point of a map in the space of initial data, the RG map, and the stability analysis becomes the analysis of the stability of the fixed point under the RG 画.

Let us fix for the Eq. (画) some (Banach) space of initial data $\Sigma$. Next, we chose a number $L>1$ and set ${ }^{(L)} y(t, x)=L^{\alpha} y\left(L^{2} t, L x\right)$, where $y$ solves Eq. (四) with the initial data $f \in \Sigma$. The RG map $\Re: \Sigma \rightarrow \Sigma$ is in this case $(\Re f)(x)=\stackrel{(L)}{y}(1, x) \stackrel{(L)}{y}(t, x)$ satisfies the equation

$$
\frac{\partial^{(L)} y(t, x)}{\partial t}=\frac{\partial^{2} \stackrel{(L)}{y}(t, x)}{\partial x^{2}}+\stackrel{(L)}{F}\left(\stackrel{(L)}{y}(t, x), \frac{\partial^{(L)} y(t, x)}{\partial x}, \frac{\partial^{2} \stackrel{(L)}{y}(t, x)}{\partial x^{2}}\right)
$$

where $\stackrel{(L)}{F}(a, b, c)=L^{2+\alpha} F\left(L^{-\alpha} a, L^{-1-\alpha} b, L^{-2-\alpha} c\right)$. The renormalization group transformation depends on $L$ and the functional form of $F$. The semi-group property of the transformation implies $\Re_{L^{n}, F}=\Re_{L, F n-1} \circ \ldots \circ \Re_{L, F_{L}} \circ \Re_{L, F}$. Letting $t=L^{2 n}$, the long time asymptotics of the solution of Eq. (四) can be obtained by iterating the fixed time problem: $y(t, x)=t^{-\alpha / 2}\left(\Re_{L^{n}, F} f\right)\left(t^{-1 / 2} x\right)$, for $t \rightarrow \infty$. 
If there is an $\alpha$ so that we can find two functions $F^{*}$ and $f^{*}$ so that $F_{L^{n}} \rightarrow F^{*}$ and $\Re_{L^{n}, F} f \rightarrow f^{*}$, so that $F^{*}$ satisfies the scale invariant equation $\dot{y}=y^{\prime \prime}+F^{*}$ and $f^{*}$ is the fixed point corresponding to this equation, defined as $\Re_{L, F^{*}} f^{*}=f^{*}$, then the asymptotics of the initial problem are given by $y\left(t, t^{-1 / 2} x\right) \sim t^{-\alpha / 2} f^{*}(x)$. Therefore the long time behavior of the differential equation is determined by the fixed point in the RG group, and the stability analysis can be also performed by studying the perturbations around this point.

\section{RENORMALIZATION GROUP TRANSFORMATIONS AND THE EQUA- TIONS OF STATE}

The energy momentum tensor of a bulk viscous cosmological fluid filling a flat FRW Universe, with line element:

$$
d s^{2}=d t^{2}-a^{2}(t)\left(d x^{2}+d y^{2}+d z^{2}\right)
$$

is given by 42, 43:

$$
T_{i}^{k}=(\rho+p+\Pi) u_{i} u^{k}-(p+\Pi) \delta_{i}^{k}
$$

where $\rho$ is the energy density, $p$ the thermodynamic pressure, $\Pi$ the bulk viscous pressure and $u_{i}$ the four velocity satisfying the condition $u_{i} u^{i}=1$. We use units so that $8 \pi G=c=1$.

The gravitational field equations, together with the continuity equation $T_{i ; k}^{k}=0$, imply

$$
\begin{aligned}
3 H^{2} & =\rho, \\
2 \dot{H}+3 H^{2} & =-p-\Pi, \\
\dot{\rho}+3(\rho+p) H & =-3 H \Pi,
\end{aligned}
$$

where $H=\dot{a} / a$ is the Hubble parameter.

The causal evolution equation for the bulk viscous pressure is given by 42, 43]

$$
\tau \dot{\Pi}+\Pi=-3 \xi H-\frac{1}{2} \tau \Pi\left(3 H+\frac{\dot{\tau}}{\tau}-\frac{\dot{\xi}}{\xi}-\frac{\dot{T}}{T}\right)
$$

where $T$ is the temperature, $\xi$ the bulk viscosity coefficient and $\tau$ the relaxation time. Eq. (11) arises as the simplest way (linear in $\Pi$ ) to satisfy the $H$ theorem ( i.e., for the entropy

production to be non-negative, $S_{; i}^{i}=\Pi^{2} / \xi T \geq 0$, where $S^{i}=e N^{i}-\frac{\tau \Pi^{2}}{2 \xi T} u^{i}$ is the entropy 
flow vector, $e$ is the entropy per particle and $N^{i}=n u^{i}$ is the particle flow vector) [23, 24]. When the the condition $\frac{T}{a^{3} H}\left|\Pi \frac{d}{d t}\left(\frac{a^{3} \tau}{\xi T}\right)\right|<<1$ holds, the additional terms to the evolution equation are negligible in comparison with $3 \xi H$, and the full equation leads to a truncated equation with reduced relaxation time and reduced bulk viscosity 43.

We also suppose that the bulk viscous cosmological fluid obeys the barotropic equation of state

$$
p=(\gamma-1) \rho, \quad 1 \leq \gamma \leq 2
$$

With this choice the conservation equation $(10)$ can be formally integrated to give

$$
\rho(t)=\frac{1}{a^{3 \gamma}}\left(\rho_{0}+\int a^{3 \gamma-1} \dot{a} \Pi d t\right),
$$

where $\rho_{0} \geq 0$ is a constant of integration.

We consider the following scale transformations:

$$
\begin{aligned}
t & \rightarrow L t, \\
a(t) & \rightarrow \stackrel{(L)}{a}(t) \equiv L^{n} a(L t),
\end{aligned}
$$

where $L>1$ is the parameter of the scale transformation and $n$ is a constant. With the use of Eqs. (14)-(15), from the Hubble parameter definition and from the field equation (9), it follows that the Hubble parameter and the energy density are scaled in the following way:

$$
\begin{aligned}
H(t) & \rightarrow \stackrel{(L)}{H}(t)=L H(L t), \\
\rho(t) & \rightarrow \stackrel{(L)}{\rho}(t)=L^{2} \rho(L t) .
\end{aligned}
$$

From equations (9) and (10) we can now easily find the scaling properties of the bulk viscous pressure and of the scale factor respectively:

$$
\begin{aligned}
& \Pi(t) \rightarrow \stackrel{(L)}{\Pi}(t)=L^{2} \Pi(L t), \\
& a(t) \rightarrow \stackrel{(L)}{a}(t)=L^{-\frac{2}{3 \gamma}} a(L t) .
\end{aligned}
$$

Eq.(11) is scale invariant with respect to transformations (14)-(19), if the relaxation time $\tau$, the bulk viscosity coefficient $\xi$ and the temperature $T$ obey the following scaling laws:

$$
\begin{aligned}
\tau(t) & \rightarrow \stackrel{(L)}{\tau}(t)=L^{-1} \tau(L t), \\
\xi(t) & \rightarrow \stackrel{(L)}{\xi}(t)=L \xi(L t), \\
T(t) & \rightarrow \stackrel{(L)}{T}(t)=L^{m} T(L t) .
\end{aligned}
$$


where $m$ is a constant. The causal evolution equation does not impose a specific scaling law for the temperature.

In order to close the system of field equations (8)-(11) we have not only to give the equation of state for $p$, but also specify $T, \tau$ and $\xi$. There are many proposals in the physical literature for equations of state for the relaxation time, temperature and bulk viscosity coefficient [25], [44, 48].

Murphy [46], Belinskii and Khalatnikov [47] and Belinskii, Nikomarov and Khalatnikov [48] have analyzed the relativistic kinetic equation for some simple cases. Their results show that in the asymptotic regions of small and large values of density the viscosity coefficients can be approximated by power functions of the energy density, with definite requirements on the exponents of these functions. For small values of the energy density it is reasonable to consider large exponents, equal, in the extreme case, to one. For large $\rho$, the power of the bulk viscosity coefficient should be considered smaller (or equal) to $\frac{1}{2}$. Hence, in the following we assume the following simple phenomenological laws [42, 43], [47, 48]:

$$
\begin{aligned}
\xi & =\alpha \rho^{s}, \\
T & =\beta \rho^{r}, \\
\tau & =\frac{\xi}{(\rho+p) c_{b}^{2}}=\alpha \rho^{s-1},
\end{aligned}
$$

where $c_{b}$ is the speed of bulk viscous perturbations, i. e., the non-adiabatic contribution to the speed of sound in a dissipative fluid without heat flux or shear viscosity [43], and $\alpha \geq 0$, $\beta \geq 0, r \geq 0$ and $s \geq 0$ are constants. Equations (22)-(25) are standard in cosmological models, also giving a simple procedure to ensure that the speed of viscous pulses does not exceed the speed of light [47, 48]. With this choice we obtain the following scaling equation for the bulk viscosity coefficient:

$$
\begin{aligned}
& \xi(t)=\alpha \rho^{s} \rightarrow \stackrel{(L)}{\xi}(t)=\alpha L^{2 s} \rho^{s}(L t), \\
& \stackrel{(L)}{\xi}(t)=L^{2 s} \xi(L t)=L \xi(L t),
\end{aligned}
$$

where we have also used Eqs. (20)-(22). From Eq.(26) it immediately follows that a scale invariant bulk viscosity coefficient must have $s=\frac{1}{2}$, and therefore the only physically acceptable behavior of the bulk viscosity coefficient is of the form $\xi \sim \rho^{1 / 2} \sim H$. In fact, this result immediately follows from Eq. (26), since the bulk viscosity coefficient has the 
same scaling transformation law as the Hubble parameter. In this way, in scale-invariant bulk cosmological models the bulk viscosity coefficient is also determined by the expansion rate of the Universe. On the other hand, $s=\frac{1}{2}$ is a case for which the gravitational field equations for a flat causal bulk viscous cosmological fluid have been intensively investigated in the physical literature [36], [37, [49, 51].

The scaling property of the relaxation time can also be obtained easily and is given by:

$$
\begin{aligned}
& \tau(t)=\alpha \rho^{s-1} \rightarrow \stackrel{(L)}{\tau}(t)=\alpha L^{2 s-2} \rho^{s-1}(L t), \\
& \stackrel{(L)}{\tau}(t)=L^{2 s-2} \tau(L t)=L^{-1} \tau(L t) .
\end{aligned}
$$

The scaling law (27) holds again only for $s=\frac{1}{2}$.

In the context of irreversible thermodynamics $p, \rho, T$ and the particle number density $n$ are equilibrium magnitudes which are related by equations of state of the form $\rho=\rho(T, n)$ and $p=p(T, n)$. From the requirement that the entropy is a state function [42], we obtain the equation $\left(\frac{\partial \rho}{\partial n}\right)_{T}=\frac{\rho+p}{n}-\frac{T}{n}\left(\frac{\partial p}{\partial T}\right)_{n}$. For the equations of state (23)-(25), this relation imposes the constraint $r=(\gamma-1) / \gamma$ [42], so that $0 \leq r \leq 1 / 2$ for $1 \leq \gamma \leq 2$, a range of values which is usually considered in the physical literature [37. Therefore we obtain the following scaling law for the temperature of the causal bulk viscous cosmological fluid obeying the state equations (23,25)

$$
\begin{aligned}
& T(t)=\beta \rho^{\frac{\gamma-1}{\gamma}} \rightarrow \stackrel{(L)}{T}(t)=\beta L^{2 \frac{\gamma-1}{\gamma}} \rho^{\frac{\gamma-1}{\gamma}}(L t), \\
& \stackrel{(L)}{T}(t)=L^{2 \frac{\gamma-1}{\gamma}} T(L t) .
\end{aligned}
$$

An alternative set of equations of state has been used by Coley, van den Hoogen and Maartens [40] to study the full causal dissipative evolution of a FRW space-time. The equations of state proposed in [40] are:

$$
\begin{aligned}
\frac{\xi}{H} & =3 \xi_{0} x^{m}, \\
\tau^{-1} & =H x^{n}, \\
x & \equiv \frac{\rho}{3 H^{2}},
\end{aligned}
$$

with $\xi_{0}, m$ and $n$ constants. By writing, with the use of Eqs. (29)-(31), the Einstein field equations as a plane autonomous system, the qualitative behavior and the evolution of the Universe can be determined. 
The scaling behaviors of the bulk viscosity coefficient and of the relaxation time are given, for the phenomenological choice of the equation of state Eqs. (29)-(31), by

$$
\begin{gathered}
\xi(t) \rightarrow \stackrel{(L)}{\xi}(t)=3 \xi_{0} L H(L t) \frac{L^{2 m} \rho^{m}(L t)}{3^{m} L^{2 m} H^{2 m}(L t)}=L \xi(L t) \\
\tau^{-1}(t) \rightarrow \stackrel{(L)}{\rightarrow} \tau^{-1}=L H(L t) \frac{L^{2 n} \rho^{n}(L t)}{3^{n} L^{2 n} H^{2 n}(L t)}=L \tau^{-1}(L t) .
\end{gathered}
$$

Therefore the set of equations of state (29)-(31) are scale invariant for all $m$ and $n$.

For a flat FRW space-time, filled with a perfect fluid obeying a $\gamma$-law barotropic equation of state, the Hubble parameter $H$ induces a natural time scale via the transformation $t \rightarrow$ $t_{H}=\frac{2}{3 \gamma} \frac{1}{H}$. The field equations are invariant with respect to this transformation, having the form $3\left(\frac{1}{a\left(t_{H}\right)} \frac{d a\left(t_{H}\right)}{d t_{H}}\right)^{2}=\rho$ and $2 \frac{d H\left(t_{H}\right)}{d t_{H}}+3 \gamma H^{2}\left(t_{H}\right)=0$. Therefore the RG analysis of the field equations for the perfect fluid can also be performed in this time variable, and the RG transformation in the Hubble time scale $t_{H} \rightarrow L t_{H}$ leads to equations which are formally similar to those obtained by using the usual cosmological time $t$. The introduction of the bulk viscous pressure generally breaks the invariance of the gravitational field equations with respect to the Hubble time. In this case, by assuming a reparametrization of the cosmological time of the form $t \rightarrow t_{0} / H, t_{0}=$ constant, the 00 component of the field equations is again invariant, but the $i i, i \neq 0$ components take the form $2 \frac{d H\left(t_{H}\right)}{d t_{H}}\left(\frac{3 \gamma t_{0}}{2}+\frac{t_{0}}{2} \frac{\Pi}{H^{2}}\right)+3 \gamma H^{2}\left(t_{H}\right)=$ $-\Pi\left(t_{H}\right)$. This equation is scale invariant only if the bulk viscous pressure is proportional to the energy density of the fluid: $\Pi \sim H^{2}=\Pi_{0} \rho, \Pi_{0}=$ constant. Then the numerical coefficient in the scaling law of the time is given by $t_{0}=2 /\left(3 \gamma+\Pi_{0}\right)$. For all other functional forms of the bulk viscous pressure, the Hubble parameter does not generate an invariant time scale for the Einstein field equations. 


\section{RENORMALIZATION GROUP EQUATIONS AND LONG TIME BEHAVIOR ANALYSIS}

Letting $t=L$, from the renormalization group transformations (14)-(20) we obtain [11]:

$$
\begin{aligned}
& a(t)=t^{\frac{2}{3 \gamma}} \stackrel{(t)}{a}(1), \\
& H(t)=t^{-1} \stackrel{(t)}{H}(1), \\
& \Pi(t)=t^{-2} \stackrel{(t)}{\Pi}(1), \\
& \xi(t)=t^{-1} \stackrel{(t)}{\xi}(1), \\
& \tau(t)=t^{(t)}(1) .
\end{aligned}
$$

To obtain the renormalization group equations we introduce the parameter of the renormalization group transformations in the form $L=e^{\theta}$. The infinitesimal transformations of $\stackrel{(L)}{a}, \stackrel{(L)}{H}$ and $\stackrel{(L)}{\Pi}$, with respect to $\theta$, are given by:

$$
\begin{aligned}
\frac{d^{(L)} a}{d \theta} & =-\frac{2}{3 \gamma} \stackrel{(L)}{a}+\frac{\partial^{(L)} a}{\partial t} \\
\frac{d \stackrel{(L)}{H}}{d \theta} & =\stackrel{(L)}{H}+\frac{\partial \stackrel{(L)}{H}}{\partial t} \\
\frac{d \stackrel{(L)}{\Pi}}{d \theta} & =2 \stackrel{(L)}{\Pi}+\frac{\partial \stackrel{(L)}{\Pi}}{\partial t} .
\end{aligned}
$$

With the use of the (scaled) gravitational field equations and of the evolution equation of the bulk viscous pressure the renormalization group equations (39)-(41) can be written as:

$$
\begin{aligned}
& \frac{d \stackrel{(L)}{a}}{d \theta}=-\frac{2}{3 \gamma} \stackrel{(L)}{a}+\stackrel{(L)}{a} \stackrel{(L)}{H} \\
& \frac{d \stackrel{(L)}{H}}{d \theta}=\stackrel{(L)}{H}+\left[-\frac{3 \gamma}{2} \stackrel{(L)}{H}^{2}-\frac{1}{2} \stackrel{(L)}{\Pi}\right]
\end{aligned}
$$

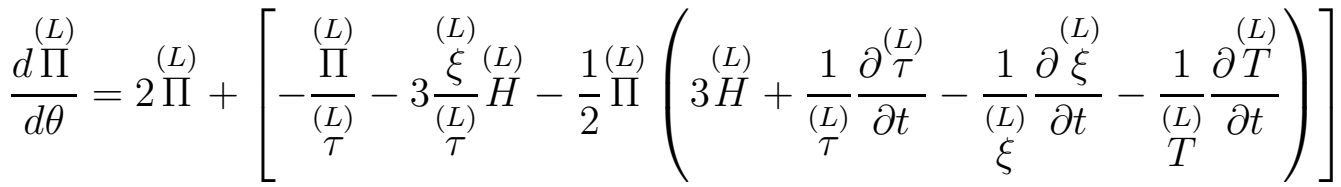

The fixed point $\left(a^{*}, H^{*}, \Pi^{*}, \xi^{*}, \tau^{*}, T^{*}\right)$ of the $\mathrm{RG}$ equations is given as a solution of the system of algebraic equations obtained by setting [11]

$$
\frac{d a^{*}}{d \theta}=0, \frac{d H^{*}}{d \theta}=0, \frac{d \Pi^{*}}{d \theta}=0 .
$$


From the flow in the RG around the fixed point we can see the long time-behavior of causal bulk-viscous fluid filled flat Universe, once the equations of state for the bulk viscosity coefficient, relaxation time and temperature are given.

As an application of the mathematical formalism presented above we shall consider the long time-behavior of the causal bulk viscous cosmological model obtained by adopting the equations of state (29)-(31), with $s=\frac{1}{2}$ and $r=\frac{\gamma-1}{\gamma}$. In this case the gravitational and causal bulk evolution equations are scale-invariant. The equations of state take the simple form $\xi=\sqrt{3} \alpha H, \tau=\frac{\alpha}{\sqrt{3} H}$ and $T=3^{r} \beta H^{2 r}$. The causal bulk viscous pressure evolution equation is

$$
\tau \dot{\Pi}+\Pi=-3 \xi H-\frac{1}{2} \tau \Pi\left[3 H-2(1+r) \frac{\dot{H}}{H}\right],
$$

while the RG equation (44) becomes

$$
\frac{d \stackrel{(L)}{\Pi}}{d \theta}=2 \prod^{(L)}+\left[-\frac{(L)}{\Pi} \underset{\tau}{(L)}-3 \frac{(L)}{{ }^{(L)}} \stackrel{(L)}{H}-\frac{1}{2} \stackrel{(L)}{\prod}\left(6 \gamma \stackrel{(L)}{H}+(1+r) \frac{(L)}{\prod_{(L)}^{H}}\right)\right] .
$$

The fixed point of the RG equations (42), (43) and (47) is defined by

$$
\begin{aligned}
& a^{*}=\left(\frac{3 \gamma^{2} \rho_{0}}{4}\right)^{1 / 3 \gamma}, H^{*}=\frac{2}{3 \gamma}, \rho^{*}=\frac{4}{3 \gamma^{2}}, \\
& \Pi^{*}=0, \xi^{*}=0, \tau^{*-1}=0 .
\end{aligned}
$$

The fixed point of the RG equations corresponds to a flat, non-viscous Friedmann Universe. In order to study the flow in the RG around the fixed point, we must study the perturbation around the fixed point. We define the perturbed quantities $\delta^{(L)} a^{2}, \delta \stackrel{(L)}{H}, \delta{\stackrel{(L)}{\Pi}, \delta^{(L)}}_{\xi}$ and $\delta \tau^{-1}$ by 11

$$
\begin{aligned}
& \stackrel{(L)}{a}=a^{*}+\delta^{(L)} a, \\
& \stackrel{(L)}{H}=H^{*}+\delta \stackrel{(L)}{H}, \\
& \stackrel{(L)}{\Pi}=\Pi^{*}+\delta \stackrel{(L)}{\Pi,} \\
& \stackrel{(L)}{\xi}=\xi^{*}+\delta \stackrel{(L)}{\xi}=\sqrt{3} \alpha \delta \stackrel{(L)}{H}, \\
& \stackrel{(L)}{\tau^{-1}}=\tau^{*-1}+\delta \tau^{-1}=\frac{\sqrt{3}}{\alpha} \delta \stackrel{(L)}{H},
\end{aligned}
$$


where the perturbations $\delta \stackrel{(L)}{a}, \delta \stackrel{(L)}{H}, \delta \stackrel{(L)}{\Pi}, \stackrel{(L)}{\xi}$ 至 and $\delta \tau^{-1}$ are assumed to be small quantities, $\delta \stackrel{(L)}{a}<<1$ etc. From the RG equations (42), (43) and (47), it follows that the perturbed quantities satisfy the following linearized differential equations

$$
\begin{aligned}
& \frac{d \delta^{(L)} a}{d \theta}=a^{*} \delta \stackrel{(L)}{H}, \\
& \frac{d \delta \stackrel{(L)}{H}}{d \theta}=-\delta \stackrel{(L)}{H}-\frac{1}{2} \delta \stackrel{(L)}{\Pi}, \\
& \frac{d \delta \stackrel{(L)}{\Pi}}{d \theta}=-\frac{4}{\gamma^{2}} \delta \stackrel{(L)}{H} .
\end{aligned}
$$

From Eqs. (55) and (56) we obtain

$$
\frac{d^{2} \delta^{(L)} a}{d \theta^{2}}+\frac{d \delta^{(L)} a}{d \theta}+\frac{a^{*}}{2} \delta \stackrel{(L)}{\Pi}=0,
$$

while Eqs. (56) and (57) give the following evolution equation for the perturbed bulk viscous pressure:

$$
\frac{d^{2} \delta \prod^{(L)}}{d \theta^{2}}+\frac{d \delta \prod^{(L)}}{d \theta}-\frac{2}{\gamma}\left(\frac{1}{\sqrt{3} \alpha}-\frac{1}{\gamma}\right) \delta \stackrel{(L)}{\Pi}=0 .
$$

The general solution of Eq. (59) is given by

$$
\delta \stackrel{(L)}{\prod}(\theta)=C_{1} e^{r_{1} \theta}+C_{2} e^{r_{2} \theta},
$$

with $C_{1}, C_{2}$ constants of integration and

$$
r_{1,2}=\frac{-1 \pm \sqrt{1+\frac{8}{\gamma}\left(\frac{1}{\sqrt{3} \alpha}-\frac{1}{\gamma}\right)}}{2} .
$$

From (61) it follows that $r_{2}<0, \forall \alpha, \gamma$ while the sign of $r_{1}$ depends on the sign of the quantity $\left(\frac{1}{\sqrt{3} \alpha}-\frac{1}{\gamma}\right)$. If $\left(\frac{1}{\sqrt{3} \alpha}-\frac{1}{\gamma}\right)>0,(\gamma>\sqrt{3} \alpha)$ then $r_{1}>0$ while for $\gamma<\sqrt{3} \alpha, r_{1}<0$.

With the use of Eq. (60), equation (58), describing the evolution of the perturbation in RG of the scale factor of the causal bulk viscous fluid filled Universe takes the form

$$
\frac{d^{2} \delta^{(L)} a}{d \theta^{2}}+\frac{d \delta^{(L)} a}{d \theta}+\frac{a^{*}}{2}\left(C_{1} e^{r_{1} \theta}+C_{2} e^{r_{2} \theta}\right)=0
$$

and has the general solution given by

$$
\delta^{(L)} a(\theta)=f_{1} e^{-\theta}+f_{2} e^{r_{1} \theta}+f_{3} e^{r_{2} \theta},
$$


where $f_{i}, i=1,2,3$ are constants of integration.

From Eq. (63) we can see the flow in the RG around the fixed point. A fixed point $y_{0}$ of a system $\dot{y}=F(y)$ is said to be stable if for every neighbourhood $U$ of $y_{0}$, there is a neighbourhood $U^{\prime} \leq U$ of $y_{0}$ such that every trajectory which passes through $U^{\prime}$ remains in $U$ as $t$ increases. The fixed point is asymptotically stable if it is stable and there is a neighbourhood $U$ of $y_{0}$ such that every trajectory passing through $U$ approaches $y_{0}$ as $t$ tends to infinity. A fixed point which is not stable is said to be unstable [53].

For $\gamma<\sqrt{3} \alpha$ the fixed point is an attractor. Then, by setting the initial profile in the vicinity of the fixed point (48)-(49), the flat causal bulk viscous Universe with bulk viscosity coefficient proportional to the Hubble parameter will approach, in the long-time limit, the flat Friedmann Universe with $a(t)=a^{*} t^{2 / 3 \gamma}$. This point is also asymptotically stable. For $\gamma>\sqrt{3} \alpha$, there is a single relevant mode. Therefore, if $\gamma>\sqrt{3} \alpha$, the space-time will deviate from the flat Friedmann geometry since there is a relevant mode $\delta a(t)=f_{2} t^{r_{1}}$. This mode corresponds to a growing mode, and therefore in this case the fixed point is asymptotically unstable.

The conditions of the asymptotic stability or instability of the solution can also be formulated in terms of the speed of sound in the cosmological fluid. Since $\sqrt{3 \alpha}=\xi / H=$ $\sqrt{3} \xi / \sqrt{\rho}=\sqrt{3 \rho} \gamma c_{b}^{2} \tau$, the condition of asymptotic stability becomes $c_{b}^{2}>1 / \sqrt{3 \rho} \tau$. With the use of the relation $\tau=\alpha \rho^{-1 / 2}$, the conditions of asymptotic stability or instability are given by $c_{b}^{2}>1 / \sqrt{3} \alpha$ and $c_{b}^{2}<1 / \sqrt{3} \alpha$, respectively.

\section{DISCUSSIONS AND FINAL REMARKS}

In the present paper we have performed an analysis of the flat homogeneous bulk viscous cosmological models by using the RG method, in the version developed for the study of asymptotics of non-linear evolution equation. For the flat FRW geometry the Einstein gravitational field equations are invariant with respect to the scale transformations of the time, energy density and Hubble parameter. Therefore it is required that the causal bulk evolution equation must also be scale invariant with respect to the same group of transformations, thus leaving invariant the full set of gravitational field and bulk viscous pressure equations. This condition leads to specific scale transformation laws for the bulk viscosity coefficient and relaxation time, and imposes strong constraints on the equations of state relating these pa- 
rameters to the energy density of the cosmological fluid. By using the property of the scale invariance of the field equations, we have shown that the most used system of equations of state, expressing the bulk viscosity parameter and relaxation time as a power of the energy density, $\xi \sim \rho^{s}, \tau \sim \rho^{s-1}$, is scale invariant for only one value of the exponent $s$. Of course this result crucially depends on the linear barotropic equation of state adopted to describe the matter content of the very early Universe. This equation of state is not satisfied in more realistic physical cases, in which, for example, the Maxwell-Boltzmann gas could provide a more appropriate description of the cosmological fluid 25].

The RG analysis also provides a powerful method for the study of the long-time asymptotics of the gravitational field equations. This can be done by the study of the flow around the fixed point of the RG group equations. For the cosmological model having the bulk viscosity coefficient proportional to the Hubble parameter $H$, the fixed point corresponds to a flat ideal (non-viscous) Friedmann type geometry. Due to the presence of viscous effects the geometry of the perturbed Universe will deviate from that of the fixed point, and the linear analysis of perturbations indicates the final state of the bulk viscous Universe. In the considered cosmological model the long-time behavior of the space- time depends on the ratio of the constant $\alpha$ relating $\xi$ to $H$ and $\gamma$.

Bulk viscous cosmological models have been investigated in the past years from many different points of view. The method of the renormalization group, as applied in a systematic manner to this class of models, can become a valuable tool for cosmologists leading to a better understanding of the nature, behavior and properties of our Universe.

\section{Acknowledgements}

The authors would like to thank to the two anonymous referees, whose comments helped to significantly improve the manuscript.

[1] K. Wilson and J. Kogut, Phys. Rep. 12C, 75 (1974)

[2] A. Lesne, Renormalization Methods: Critical Phenomena, Chaos, Fractal Structures, Chichester: John Wiley, (1998)

[3] M. Salmhofer 1998. Renormalization: an Introduction, Berlin: New York, Springer, (1998) 
[4] J. Bricmont, A. Kupiainen and G. Lin, Commun. Pure Appl. Math. 47, 893 (1994)

[5] L . Y. Chen, N. Goldenfeld and Y. Oono, Phys. Rev. E54, 376 (1996)

[6] S.-I. Ei, K. Fujii and T. Kunihiro, Annnals Phys. 280, 236 (2000)

[7] T. Koike, T. Hara and S. Adachi, Phys. Rev. Lett. 74, 5170 (1995)

[8] T. Hara, T. Koike and S. Adachi, gr-qc/9607010 (1996)

[9] M. Carfora and K. Piotrkowska, Phys. Rev. D52, 4393 (1995)

[10] Y. Sata, T. Kobayashi, K. Maeda, T. Kurokawa, M. Morikawa and A. Nakamichi, Phys. Rev. D58, $043502(1998)$

[11] O. Iguchi, A. Hosoya and T. Koike, Phys. Rev. D57, 3340 (1998)

[12] Y. Nambu and Y. Y. Yamaguchi, Phys. Rev. D60, 104011 (1999)

[13] Y. Nambu, Phys. Rev. D62, 044013 (2000)

[14] E. A. Calzetta, B. L. Hu and F. D. Mazzitelli, Phys. Repts. 352, 459 (2001)

[15] M. Reuter and F. Saueressig, Phys. Rev. D65, 065016 (2002)

[16] A. Bonnano and M. Reuter, Phys. Lett. B527, 9 (2002)

[17] I. L. Shapiro and J. Sola, JHEP 0202, 006 (2002)

[18] C. W. Misner, Phys. Rev. Lett. 19, 533 (1966)

[19] L. P. Chimento and A. Jakubi, Phys. Lett. A212, 320 (1996)

[20] C. Eckart, Phys. Rev. 58, 919 (1940)

[21] L. D. Landau and E. M. Lifshitz, Fluid Mechanics, Oxford: Butterworth Heinemann (1987)

[22] W. Israel, Ann. Phys. (NY) 100, 310 (1976)

[23] W. Israel and J. M. Stewart, Phys. Lett. A58, 213 (1976)

[24] W. A. Hiscock and L. Lindblom, Ann. Phys. (NY) 151, 466 (1989)

[25] W. A. Hiscock and J. Salmonson, Phys. Rev. D43, 3249 (1991)

[26] W. A. Hiscock and L. Lindblom, Phys. Rev. D35, 3723 (1987)

[27] W. Zimdahl, Phys. Rev. D53, 5483 (1996)

[28] W. Zimdahl, D. Pavon and R. Maartens, 1997 Phys. Rev. D55, 4681 (1997)

[29] W. Zimdahl, Phys. Rev. D57, 2245 (1998)

[30] R. Maartens and V. Mendez, Phys. Rev. D55, 1937 (1997)

[31] L. P. Chimento, A. S. Jakubi, V. Mendez and R. Maartens, Class. Quantum Grav. 14, 3363 (1997)

[32] L. Herrera and J. Martinez, Class. Quantum Grav. 15, 407 (1998) 
[33] L. Herrera and J. Martinez, Class. Quantum Grav. 14, 2697 (1997)

[34] M. Govender, S. D. Maharaj and R. Maartens, Class. Quantum Grav. 15, 323 (1998)

[35] R. Maartens and J. Triginer, Phys. Rev. D58, 123507 (1998)

[36] D. Pavon, J. Bafaluy and D. Jou, Class. Quantum Grav. 8, 347 (1991)

[37] L. P. Chimento and A. S. Jakubi, Class. Quantum Grav. 14, 1811 (1997)

[38] T. Harko and M. K. Mak, Int. J. Theor. Phys. 38, 1573 (1999)

[39] R. Maartens and J. Triginer, Phys. Rev. D56, 4640 (1997)

[40] A. A. Coley, R. J. van den Hoogen and R. Maartens, Phys. Rev. D54, 1393 (1996)

[41] V. Romano and D. Pavon, Phys. Rev. D47, 1396 (1993)

[42] R. Maartens, Class. Quantum Grav. 12, 1455 (1995)

[43] R. Maartens, astro-ph/9609119 (1996)

[44] K. Lake, Phys. Rev. D26, 518 (1982)

[45] M. Zakari and D. Jou, Phys. Rev. D48, 1597 (1993)

[46] G. L. Murphy, Phys. Rev. D8, 4231 (1973)

[47] V. A. Belinskii and I. M. Khalatnikov, Sov. Phys. JETP 42, 205 (1975)

[48] V. A. Belinskii, E. S. Nikomarov and I. M. Khalatnikov, Sov. Phys. JETP 50, 213 (1979)

[49] M. K. Mak and T. Harko, Gen. Rel. Grav. 30, 1171 (1998)

[50] M. K. Mak and T. Harko, J. Math. Phys. 39, 5458 (1998)

[51] M. K. Mak and T. Harko, Gen. Rel. Grav. 31, 273 (1999)

[52] M. K. Mak and T. Harko, Australian J. Phys. 52, 659 (1999)

[53] W. Walter, Ordinary differential equations, New York, Springer, (1998) 